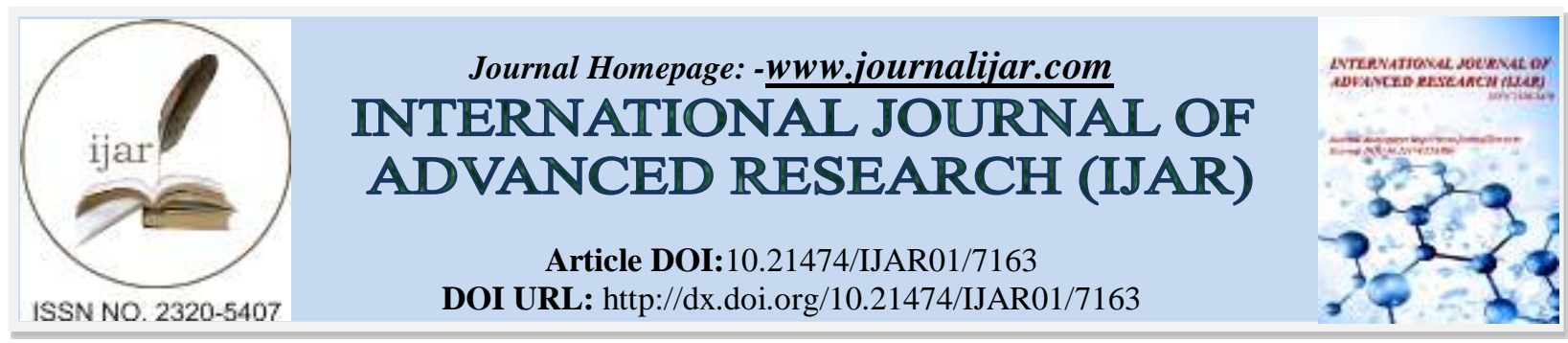

RESEARCH ARTICLE

\title{
ROLE OF SYNTAX SCORE AND EURO SCORE ON THE OUTCOME OF EGYPTIAN PATIENTS UNDERGOING CORONARY ARTERY BYPASS GRAFTS.
}

Tarek sayed Mohamed M.B.B.Ch, Yousry El-saied MD, Yousry Shaheen MD, Ibrahim Kasb MD, Ayman M. Shaalan MD, Moataz Rezk MD, Sameh Elameen MD, Ahmed Mostafa Omran MD.

\section{Manuscript Info}

Manuscript History

Received: 21 March 2018

Final Accepted: 23 April 2018

Published: May 2018

Keywords:-

CABG; SYNTAX; EURO Score.

\section{Abstract}

Background: Coronary heart disease (CHD) is a major cause of death and disability in developed countries, surgical revascularization of coronary stenosis with coronary artery bypass grafting is ideal for certain patients, despite technological advancements, open-heart operations still carry a risk of mortality and morbidity. To aid in the selection of patients for cardiac surgery, several risk scoring systems have been developed during the last decades. Risk models are used for two main reasons. The first is that a risk model such as Euro SCORE allows the calculation of the risk of death, The SYNTAX score is used to evaluate coronary artery complexity and is well correlated with early and long-term outcomes after CABG.

Objective: Identify accuracy of SYNTAX score Vs EURO score in prediction of mortality and morbidity of CABG patients in Egyptian population

Patients and Methods: prospective observational study, done at the National Heart Institute of Egypt, Amiri hospital and Adan hospital in Kuwait in the period from July 2016 till December 2017. We collected demographic data, preoperative, operative, and postoperative data. Inclusion criteria: age: from 30-80, isolated CABG without other cardiac diseases, elective cases .Exclusion criteria: emergency cases, combined ischemic and valvular cases even significant ischemic Mitral regurge and redo cases. The sheet included preoperative data regarding age, sex , weight ,DM , HTN , COPD , Liver cell failure, renal cell failure, previous hospital admission, pre and post-operative echo data, history of bleeding, history of blood transfusion, monitoring the pre and post hospital complications and also history of drug administration like ASA. The surgical procedures were performed according to the Institute and hospitals protocol. Postoperative data including hospital, ICU stay, chest tubes drainage, blood products transfusion, reexploration for bleeding and surgical site infection and any other undesired events.

Results: 70 males with age ranges between 40 and 72 years. 55 smokers, 42 diabetic on insulin 20 COPD, 10 chronic renal impairment, 5 previous CVA, 45 have dyslipidemia, same number hypertensive.

Operative and postoperative: 10 patients needed IABP, 5 had heart failure, 5 had postoperative MI, 5 reopened for re-exploration, 10 have 
superficial wound infect, 20 patients of them have postoperative arrhythmias while mortality was $5 \%$.

Conclusion: The study approved that both EURO SCORE II and SYNTAX SCORE II are accurate in the prediction of the outcome of CABG and prediction of high risk patients and is more liable for detection of morbidity and mortality and complications, however EURO score is more accurate.New score combining anatomical variant as SYNTAX score plus pathophysiological score as euro score will probably carry higher specificity and sensitivity.

Copy Right, IJAR, 2018,. All rights reserved.

\section{Introduction:-}

It is well known that a major cause of death and disability in developed countries is coronary heart disease (CHD). Although mortality rates worldwide related to coronary artery disease have declined over the past four decades, yet it remains responsible for about one-third or more of all deaths in individuals over age 35 (1-3).

Surgical management for coronary stenosis with coronary artery bypass grafting (CABG) is ideal for certain patients, especially those with three-vessel who have historically been treated with this approach, although some trials question whether multi-vessel stenting may be safer as Optimal Management of Multi-vessel disease (FREEDOM) trial.(4)

Patients with diabetes or those with severe LV systolic dysfunction have improved outcomes with surgical revascularization compared with PCI. Also, when the left main coronary artery or a left main equivalent (proximal left anterior descending and proximal circumflex) is involved, CABG is recommended. (5)

Despite technological advancements, open-heart operations still carry a risk of mortality and morbidity. To aid in the selection of patients for cardiac surgery, several risk scoring systems have been developed during the last decades. These aim to estimate the risk of peri-operative death, based on the occurrence of different risk factors. From other side operative mortality and morbidity is now increasingly used as an indicator of the quality of cardiac surgery, however to make an accurate comparison between different institutions or surgeons, mortality data must be adjusted to the risk profiles of the patients. (6)

Risk models are used for two main reasons. The first is that a risk model such as Euro SCORE allows the calculation of the risk of death before undertaking a heart operation. This is important as it serves to guide the clinician and the patient about the advisability of an operation by helping to weigh the risk against the benefits. The second is as a method of quality control. By calculating the expected risk of death for a population of patients having certain heart operations, this can be compared with the number of actual deaths. This comparison can be used as a measure of the quality of the performance of the hospital, unit or surgeon. (7)

One of the most prominent studies, the SYNTAX trial, compared multi-vessel percutaneous coronary intervention (PCI), to the coronary artery bypass graft (CABG). The results of this randomized study demonstrated that patients who had SYNTAX scores >33 appeared to do much better with bypass surgery than those with lower SYNTAX scores, in whom PCI was just as good for major adverse cardiac events, with lowering of the stroke rates. (8)

We evaluated the usefulness of the combination of European System for Cardiac Operative Risk Evaluation score(Euro SCORE II) and SYNTAX between percutaneous intervention with drug-eluting stents and cardiac surgery (SYNTAX) score in predicting risks associated with early and late outcomes after coronary artery bypass grafting (CABG). (9)

Euro SCORE II still contains no information regarding anatomy or extent of coronary artery disease, and few studies provide data regarding a relationship between Euro SCORE II and late outcomes after coronary artery bypass grafting (CABG). (10) 
SYNTAX trial, has been used mainly for preprocedural evaluation in patients undergoing PCI .The SYNTAX score is used to evaluate coronary artery complexity and is well correlated with early and long-term outcomes after PCI however, the relationship between SYNTAX score and outcomes after CABG is still debated (9)

\section{Patients and Methods:-}

This study is a prospective non randomized observational study, done at the National Heart Institute of Egypt, Amiri hospital and Adan hospital in Kuwait in the period from July 2016 till December 2017. We collected demographic data, preoperative, operative, and postoperative data from 100 consecutive patients. Risk assessment was done using Euro score II and Syntax score 2 .

The inclusion criteria was Age: from 30-80 years patients undergoing isolated CABG without other cardiac diseases (valvular or congenital) on elective basis while exclusion criteria was emergency case, combined ischemic and valvular cases even significant ischemic Mitral regurge and redo cases.

Data collection included preoperative data regarding age, sex, weight, Diabetes mellitus, hypertension, chronic obstructive pulmonary disease, Liver cell failure, renal cell failure, previous hospital admission, pre and postoperative echo data, history of bleeding, history of blood transfusion, monitoring the pre and post hospital complications and also history of drug administration like ASA. Clopidogrel was stopped for all of the patients minimum 4 days before operation and replaced by Low molecular weight Heparin. The surgical procedures were performed according to the Institute and hospitals protocol. Postoperative data included hospital, ICU stay, chest tubes drainage, blood products transfusion, re-exploration for bleeding and surgical site infection and any other undesired events.

Surgical techniques was conventional cardiac surgery using bypass technique which was done for all patients with the exception of 10 patients who were done using the off pump technique. All the patients were done by surgical protocol through median sternotomy incision not minimal invasive incision, all the patients were given heparin in a dose of 300 units per $\mathrm{Kg}$, received blood cardioplegia given by antegrade and retrograde techniques regularly every 20 minutes, the on pump patients had been done by aortic cannulation and caval cannulation, given hot shots on weaning from the bypass, protamine sulfate given for all of the patients, the grafts were left internal mammary in all of the patients and other grafts were saphenous vein grafts, radial artery grafts and right mammary grafts according to each case, the saphenous veins and radial arteries had been harvested by endoscopic technique in 40 cases and by open technique in 55 cases and 5 patients by single graft (the internal mammary to LAD), proper hemostasis done, 2 pacemakers wires inserted and closure was by stainless steel wires and surgivac inserted for the patients done by the endoscopic technique in the site of the harvesting for proper drainage.

\section{Statistical analysis:-}

The collected data were tabulated and analyzed using SPSS version 16 software (Spss Inc, Chicago, ILL Company). Categorical data were presented as number and percentages while quantitative data were expressed as mean \pm standard deviation, median and range. Fisher's exact test (FET), and McNemer's test were used to analyze categorical variables. Quantitative data were tested for normality using Kolomogrov Smirnove test, assuming normality at $\mathrm{P}>0.05$, using Paired " $\mathrm{t}$ " test for matched variables, if normally distributed, or Man Whitney $U$ test for independent variables if not normally distributed. ROC curve was used to determine cutoff value of the studied scores with optimum sensitivity and specificity in prediction of outcome. The accepted level of significance in this work was stated at 0.05 ( $\mathrm{P}<0.05$ was considered significant, $\mathrm{P}$ value $>0.05$ is non-significant and $\mathrm{P} \leq 0.001$ is highly significant.

\section{Results:-}

The study included 100 patients 70 males and 30 females with age ranging between 40 and 72 years, 55 of them are smokers, (25 cigarettes smokers for more than 10 years around one box per day while 10 of them for less than 10 years and 20 of them are shisha smokers for more than 10 years usually once per day). 65 patients are diabetics, (42 are insulin dependent and 23 are non-insulin dependent) 45 patients of them have positive family history ( table 1).

Regarding preoperative comorbidities 20 patients was known to have COPD, and they were on anti-asthmatic drugs like bronchodilators and corticosteroids. 10 patients have chronic renal impairment, on regular dialysis, urine 
output calculated for all of them total input and output for all of them in the ICU and they continued dialysis directly after the operations ( table 2).

45 patients known to be hypertensive and 45 patients were having dyslipidemia. 45 patients had preoperative MI, on the other hand 5 patients of them have preoperative CVA, (table 2).

The mean SYNTAX score was 25.5 while the mean Euro score was 0.98 .

Intra-Operative And Post-Operative Events And Complications:-

10 patients needed intra-aortic balloon pump, 5 patients were reopened for re-exploration, ( 2 of them due to tamponade and 3 of them due to bleeding from the chest tubes more than $1200 \mathrm{ml}$ within the first 3 hours). 5 patients had post-operative MI, diagnosed by ECG changes and elevated cardiac enzymes and new resting segmental wall motion abnormalities. 5 patients had postoperative heart failure, 10 patients had new renal failure, 5 patients had post-operative liver cell failure, 20 patients had postoperative arrhythmias (the commonest was atrial fibrillation), 5 of them returned to sinus rhythm. 10 patients of them had superficial wound infection, 5 patients had deep wound infection, 2 of them needed rewiring (table 3) Hospital mortality:

5 patients died post-operatively, 2 of them failed from weaning from the ventilator with past history of COPD in the ICU, 2 from heart failure and low ejection fraction post operatively and failed from weaning from the inotropes and one from deep wound infection (table 4).

On focusing on complication according to SYNTAX categorization it was found to be 2 in low SYNTAX, 4 in intermediate group and 19 patients in high SYNTAX group (table 5).

On the other hand Euro score also showed high sensitivity to predict the complication where $100 \%$ of the patients scoring 3 to 5 had complications. (table 6).

This was the same with the mortality where all mortality was in the high SYNTAX and at the same time high EURO score group.

On statistical analysis for both scores as predictors for morbidity and mortality SYNTAX score was found to be sensitive while EURO score is highly sensitive. (table 7)

On studying mortality and morbidity related to isolated risk factors, female sex, smoking, CCS, NYHA class, hypertension, dyslipidemia, preoperative MI, Cerebro-vascular strokes, and chronic renal impairment were found to be sensitive.

Socio-demographic characters of the studied sample:

\begin{tabular}{|l|l|l|l|}
\hline \multicolumn{2}{|l|}{ Variable } & No. $(\mathbf{N}=\mathbf{1 0 0})$ & \% (100\%) \\
\hline \multirow{3}{*}{ Age (ys) } & Mean \pm SD & $57.5 \pm 8.5$ & \multicolumn{2}{|l|}{} \\
\cline { 2 - 4 } & Range & $40-72$ & 70.0 \\
\hline \multirow{3}{*}{ Smoking } & Male & 70 & 30.0 \\
\cline { 2 - 4 } & Female & 30 & 55.0 \\
\hline \multirow{2}{*}{ DM } & Yes & 55 & 45.0 \\
\cline { 2 - 4 } & No & 45 & 65.0 \\
\hline \multirow{3}{*}{ Hypertension } & Yes & 65 & 35.0 \\
\cline { 2 - 4 } & No & 35 & 45.0 \\
\hline \multirow{2}{*}{ Dyslipidemia } & Yes & 45 & 55.0 \\
\cline { 2 - 4 } & No & 55 & 45.0 \\
\hline \multirow{2}{*}{ Family history } & Yes & 45 & 55.0 \\
\cline { 2 - 4 } & No & 55 & 45.0 \\
\hline
\end{tabular}


Table 1:-The mean and range in numbers and percentage for age, sex, smoking, DM (Diabetes Mellitus), hypertension, dyslipidemia, family history.

\begin{tabular}{|c|c|c|c|}
\hline \multicolumn{2}{|l|}{ Variable } & No. $(\mathrm{N}=100)$ & $\%(100 \%)$ \\
\hline \multirow[t]{3}{*}{ CCS } & CCS 2 & 35 & 35.0 \\
\hline & CCS 3 & 50 & 50.0 \\
\hline & CCS 4 & 15 & 15.0 \\
\hline \multirow[t]{3}{*}{ NYHA } & NYHA 1 & 45 & 45.0 \\
\hline & NYHA 2 & 30 & 30.0 \\
\hline & NYHA 3 & 25 & 25.0 \\
\hline \multirow[t]{2}{*}{ Preoperative MI } & Yes & 45 & 45.0 \\
\hline & No & 55 & 55.0 \\
\hline \multirow[t]{2}{*}{ Preoperative CVA } & Yes & 5 & 5.0 \\
\hline & No & 95 & 95.0 \\
\hline \multirow[t]{2}{*}{ COPD } & Yes & 20 & 20.0 \\
\hline & No & 80 & 80.0 \\
\hline \multirow[t]{2}{*}{ CRI } & Yes & 10 & 10.0 \\
\hline & No & 90 & 90.0 \\
\hline \multirow{2}{*}{$\begin{array}{ll}\text { Extra } & \text { cardiac } \\
\text { Vasculopathy } & \end{array}$} & Yes & 0 & 0.0 \\
\hline & No & 100 & 100.0 \\
\hline \multirow[t]{5}{*}{ No of diseased vessels } & One & 5 & 5.0 \\
\hline & Two & 10 & 10.0 \\
\hline & Three & 55 & 55.0 \\
\hline & Four & 25 & 25.0 \\
\hline & Five & 5 & 5.0 \\
\hline \multirow[t]{2}{*}{ Previous PCI } & Yes & 5 & 5.0 \\
\hline & No & 95 & 95.0 \\
\hline
\end{tabular}

Table 2:-The mean and range in numbers and percentage for CCS" Canadian cardiovascular society classification", NYHA" New York Heart Functional Classification", preoperative myocardial infarction, preoperative cerebrovascular accident, COPD chronic obstructive pulmonary disease, CRI chronic renal impairment, Extra Cardiac Vasculopathy, number of diseased vessels, PCI previous coronary intervention).

\begin{tabular}{|c|c|c|c|}
\hline \multicolumn{2}{|l|}{ Variable } & No. $(\mathrm{N}=100)$ & $\%(100 \%)$ \\
\hline \multirow[t]{2}{*}{ Re-exploration } & Yes & 5 & 5.0 \\
\hline & No & 95 & 95.0 \\
\hline \multirow[t]{2}{*}{ Postoperative MI } & Yes & 5 & 5.0 \\
\hline & No & 95 & 95.0 \\
\hline \multirow[t]{2}{*}{ Postoperative HF } & Yes & 5 & 5.0 \\
\hline & No & 95 & 95.0 \\
\hline \multirow[t]{2}{*}{ Postoperative renal failure } & Yes & 10 & 10.0 \\
\hline & No & 90 & 90.0 \\
\hline \multirow{2}{*}{$\begin{array}{l}\text { Postoperative } \\
\text { CVA }\end{array}$} & Yes & 0 & 0.0 \\
\hline & No & 100 & 100.0 \\
\hline \multirow[t]{2}{*}{ Postoperative LCF } & Yes & 5 & 5.0 \\
\hline & No & 95 & 95.0 \\
\hline \multirow[t]{2}{*}{ Arrhythmias } & Yes & 20 & 20.0 \\
\hline & No & 80 & 80.0 \\
\hline \multirow{2}{*}{$\begin{array}{ll}\text { Superficial } & \text { wound } \\
\text { infection } & \end{array}$} & Yes & 10 & 10.0 \\
\hline & No & 90 & 90.0 \\
\hline \multirow[t]{2}{*}{ Deep wound infection } & Yes & 5 & 5.0 \\
\hline & No & 95 & 95.0 \\
\hline \multirow[t]{2}{*}{ ICU stay (hours) } & Mean \pm SD & \multicolumn{2}{|l|}{$83.7 \pm 30.9$} \\
\hline & Range & \multicolumn{2}{|l|}{$56-206$} \\
\hline \multirow[t]{2}{*}{ Hospital stay (days) } & Mean \pm SD & \multicolumn{2}{|l|}{$7.55 \pm 2.45$} \\
\hline & Range & \multicolumn{2}{|l|}{$6-15$} \\
\hline
\end{tabular}


Table 3:-The mean and range in numbers and percentage for Re-exploration, post-operative myocardial infarction, post-operative heart failure, post-operative renal failure, post-operative cerebrovascular accident, post-operative liver cell failure, Arrhythmias, Superficial wound infection, Deep wound infection, ICU stay in hours, Hospital stay in days .

\begin{tabular}{|l|l|l|l|}
\hline \multicolumn{2}{|l|}{ Variable } & No. $(\mathbf{N}=\mathbf{1 0 0})$ & $\mathbf{\%}(\mathbf{1 0 0 \%})$ \\
\hline \multirow{2}{*}{ Mortality } & Yes & 5 & 5.0 \\
\cline { 2 - 4 } & No & 95 & 95.0 \\
\hline
\end{tabular}

Table 4:-Numbers and percentage for the number of Mortality cases post-operative

\begin{tabular}{|l|l|l|l|l|}
\hline & & \multicolumn{2}{l|}{ COPMLICATION } & \multirow{2}{*}{ Total } \\
\hline \multirow{2}{*}{ Low </=18 } & & No & Yes & 10 \\
& Count & 8 & 2 & $100.0 \%$ \\
\cline { 2 - 5 } & \% within SYCBAGs & $80.0 \%$ & $20.0 \%$ & 50 \\
\hline \multirow{2}{*}{ Intermediate $>\mathbf{1 8 - 2 7}>\mathbf{2 7}$} & Count & 46 & 4 & $100.0 \%$ \\
\cline { 2 - 5 } & \% within SYCBAGs & $92.0 \%$ & $8.0 \%$ & 40 \\
\cline { 2 - 5 } & Count & 21 & 19 & $100.0 \%$ \\
\hline
\end{tabular}

FET $=18.5$

$\mathbf{P}<0.001$ (HS)

Table 5:-Numbers and percentage for complications according to the Risk grades in the SYNTAX score in CABG.

\begin{tabular}{|c|c|c|c|c|c|}
\hline & & & \multicolumn{2}{|c|}{ COPMLICATION2 } & \multirow[t]{2}{*}{ Total } \\
\hline & & & No & Yes & \\
\hline \multirow[t]{4}{*}{ EURO } & \multirow[t]{2}{*}{ Low 0-2 } & Count & 75 & 20 & 95 \\
\hline & & \% within EUROs & $78.9 \%$ & $21.1 \%$ & $100.0 \%$ \\
\hline & \multirow[t]{2}{*}{ Medium 3-5 } & Count & 0 & 5 & 5 \\
\hline & & \% within EUROs & $.0 \%$ & $100.0 \%$ & $100.0 \%$ \\
\hline \multirow{2}{*}{\multicolumn{2}{|c|}{ Total }} & Count & 75 & 25 & 100 \\
\hline & & \% within EUROs & $75.0 \%$ & $25.0 \%$ & $100.0 \%$ \\
\hline
\end{tabular}

FET was used $\mathbf{P}=0.001(\mathrm{HS})$

Table 6:-Numbers and percentage for complications according to the Risk grades in the EURO score for surgery.

\begin{tabular}{|l|l|l|l|l|l|l|l|l|}
\hline Score & Sens\% & Spec\% & PPV\% & NPV\% & $\begin{array}{l}\text { Accuracy } \\
\%\end{array}$ & AUC & 95\% CI & P \\
\hline $\begin{array}{l}\text { Syntax } \\
\text { CABG } \\
\mathbf{2 8 . 8}\end{array}$ & $100 \%$ & $68.4 \%$ & $14.2 \%$ & $100 \%$ & $70 \%$ & 0.832 & $0.71-0.95$ & $\begin{array}{l}0.013 \\
(\text { S) }\end{array}$ \\
\hline $\begin{array}{l}\text { EURO } \\
\geq \mathbf{1 . 0 6}\end{array}$ & $100 \%$ & $100 \%$ & $100 \%$ & $100 \%$ & $100 \%$ & 1.000 & $1.0-1.0$ & $<0.001$ \\
$($ HS $)$
\end{tabular}

Table 7:-Specificity and sensitivity for both scores SYNTAX

\section{Discussion:-}

The recent studies showed that the high EURO SCORE II $(\geq 1.41)$ and high SYNTAX SCORE II ( $\geq 32)$ were nondependable factors for prediction of major complications for isolated CABG. In our study we found that the patients with the high EURO SCORE II and high SYNTAX SCORE II had the high risk for the early major post-operative complications. (11)

The study had been done on 100 patients 70 males and 30 females aged between 42 and 70 years. All the patients subjected to EURO SCORE II and SYNTAX SCORE II and all of them did CABG. The mortality rate was $5 \%$, all in the female group, that showed that the female gender playing a role in the outcome of CABG results and all this patients were having high EURO SCORE II. This result coincided with the study that had been done by Niv Ad et al.(11)

The cases evaluated according to CCS (Canadian Cardiovascular Society classification) and 35 of them were class 2 , 50 them were class 3 and 15 of them were class 4 . All the cases of mortality were in class 4 with no mortality in 
class 2 and 3. The percentage was $15 \%$ from class 4 and $5 \%$ from the total cases. This is indicating that the high CCS class represents a very important indicator for the outcome and complications among the patients and approve that they have an impact on the prediction of EURO SCORE II and SYNTAX SCORE II.This coincided with the study that had been held by Muhammad Sher et al., that showed that good outcome better with CABG than PCI for the patients with high CCS class with increasing the mortality rate for the patients with class 4 CCS and better outcome with less complications with the surgery than PCI .(12)

The study included 65 patients known to be diabetic with mortality among the diabetic included 5 patients.on the other hand 45 patients known to be hypertensive and on regular medications prior to the procedures. The mortality were 5 patients all of them were hypertensive with positive statistical significance that showed that the hypertension play important role on the outcome of the results and the role of it in the prediction of the outcome of the EURO SCORE II, like other studies that showed that the hypertension and DM had many complications and affect the outcome and mortality among the patients especially with the left main patients. (13)

In the study there were 45 patients had pre-operative myocardial infarction. All the mortality cases were between the cases of pre-operative myocardial infarction which included 5 cases. This clarify that the pre-operative myocardial infarction playing a major role in the outcome of CABG and coincided with the prediction of complications and mortality with high EURO SCORE II and SYNTAX SCORE II. This matched with the study done by Kamal et al. The study proved that the pre-operative myocardial infarction has an important role in the outcome of CABG and co-related with prediction of the outcome of EURO SCORE II and SYNTAX SCORE II.The same is with CVA were all the 5 cases that had CVA ( Cerebrovascular Accident ) died as other studies showed.(13, 14)

The mortality cases were in the female patients that proved that several risk factors shared in their mortality as gender, chronic renal impairment, COPD and high EURO SCORE II and SYNTAX SCORE II. These cases were on regular dialysis pre-operatively, This is the same as other studies targeting same issue. (15)

All cases of mortality was found to have high SYNTAX score as well as morbidity where there mean SYNTAX was 32.7, On comparing between sensitivity and specificity of both scores, we found that SYNTAX score was sensitive while EURO score was highly sensitive; also EURO score is more specific than SYNTAX score.

\section{Study limitations:-}

A greater number of patients are needed to increase the effectiveness of the study, sharing of more centers may emphasize the point of this study and make it more clear.

\section{Conclusion and recommendations:-}

There are several scores that used in prediction of the outcome of CABG and in the prediction of the cases which may be associated with morbidity and mortality.The most important of these scores EURO SCORE II, SYNTAX SCORE II.The study approved that both EURO SCORE II and SYNTAX SCORE II are accurate in the prediction of the outcome of CABG and prediction of high risk patients and is more liable for detection of morbidity and mortality and complications, however EURO score is more accurate. New score combining anatomical variant as SYNTAX score plus pathophysiological score as EURO score will probably carry higher specificity and sensitivity. 


\section{References:-}

1. Rosamond W, Flegal K, Furie K, et al. Heart disease and stroke statistics 2008 update: a report from the American Heart Association Statistics Committee and Stroke Statistics Subcommittee. Circulation 2008; 117:e25, Epidemiology of coronary heart disease and acute coronary syndrome.

2. Nichols M, Townsend N, Scarborough P, Rayner M. Cardiovascular disease in Europe 2014: epidemiological update. Eur Heart J 2014; 35:2950.

3. Benjamin EJ, Blaha MJ, Chiuve SE, et al. Heart Disease and Stroke Statistics-2017 Update: A Report From the American Heart Association. Circulation 2017; 135:e146.

4. Afnan R Tariq, Ailin Barseghian El-Farra, The FREEDOM Trial: Revascularization in Diabetics with Multivessel Disease. A Population-Based Evaluation of Outcomes ACC 2016/05/12/08/27.

5. Michael E. Farkouh, Michael Domanski, Lynn A. Sleeper, George Dangas, , Michael Mack, May Yang, et al . Strategies for Multivessel Revascularization in Patients with Diabetes, N Engl J Med 2012;367:2375-84.

6. Sidebotham, David, Andrew McKee, Jerrold Levy, Michael Gillham. Cardiothoracic Critical Care. Elsevier Health Sciences. P.611. ISBN 978-0-7506-7572-7.

7. Roques F, Nashef SA, Michel P, Gauducheau E, de Vincentiis C, Baudet E, et al. Risk factors and outcome in European cardiac surgery: analysis of the EuroSCORE multinational database of 19030 patients. Eur J Cardiothorac Surg. 1999 Jun;15(6):816-22; discussion 822-3.

8. Kappetein AP. Erasmus Medical Center, Rotterdam, The Netherlands. On behalf of the SYNTAX investigators. The 3- year outcomes of the SYNTAX trial. 24TH EACTS annual meeting, Geneva, Switzerland, 11e14 September 2010. http://www.medscape.com/viewarticle/728416.

9. ToshihiroFukui*, Tomoya Uchimuro and Shuichiro Takanashi. EURO SCORE II with SYNTAX SCORE to assess risks of coronary artery bypass grafting outcomes Eur J Cardiothorac Surg. 2015 Jan;47(1):66-71.

10. Ahmed Mostafa Omran. Effect of the type of stent on results of coronary artery bypass grafting in multivessel disease patients, JECST 24 (2016) 286-292.

11. Niv Ad Scott D. Barnett Alan M. Speir. The performance of the EuroSCORE and the Society of Thoracic Surgeons mortality risk score: the gender factor Inter. CardioVascular and Thoracic Surgery, Volume 6, Issue 2, 192-195.

12. Muhammad Sher-i-Murtaza,1 Mirza Ahmad Raza Baig,and Hafiz Muhammad Azam Raheel. Early outcome of Coronary Artery Bypass Graft Surgery in patients with significant Left Main Stem stenosis at a tertiary cardiac care center Pak J Med Sci. 2015 Jul-Aug; 31(4): 909-914.

13. Marzia Leacche, John G. Byrne, Natalia S. Solenkova, Brendan Reagan, Tahir I. Mohamed, Joseph L. Fredi, et al. Comparison of 30-day outcomes of coronary artery bypass grafting surgery verus hybrid coronary revascularization stratified by SYNTAX and EUROSCORE The Journal of Thoracic and Cardiovascular Surgery Volume 145, Issue 4, April 2013, Pages 1004-1012.

14. Yasser A. Kamal, Shady E. M. Al-Elwany, Ahmed M. F. Ghoneim, Ahmed M. K. El-Minshawy. Predictors of adverse effects after coronary artery bypass grafting in patients with reduced left ventricular ejection fraction. JESCTS Volume 25, Issue 1, Pages 20-27.

15. Stuart J. Heada, Michael J. Mackb, David R. Holmes Jrc, Friedrich W. Mohrd, Marie-Claude Moricee, Patrick W. Serruysf and A. Pieter Kappeteina, Incidence, predictors and outcomes of incomplete revascularization after percutaneous coronary intervention and coronary artery bypass grafting: a subgroup analysis of 3-year SYNTAX data. European Journal of Cardio-Thoracic Surgery 41 (2012) 535-541. 